\title{
Lidil
}

Revue de linguistique et de didactique des langues

$62 \mid 2020$

Recherches actuelles en didactique du lexique : avancées, réflexions, méthodes

\section{Monique De Mattia-Viviès, Leçons de grammaire anglaise, de la recherche à l'enseignement} 3 vol., Aix-en-Provence, Presses universitaires de Provence, coll. « Manuels », 2018 et 2019, 1807 p.

\section{Samia Ounoughi}

\section{(2) OpenEdition}

Édition électronique

URL : http://journals.openedition.org/lidil/8257

DOI : $10.4000 /$ lidil.8257

ISSN : 1960-6052

Éditeur

UGA Éditions/Université Grenoble Alpes

Édition imprimée

ISBN : 978-2-37747-226-0

ISSN : $1146-6480$

\section{Référence électronique}

Samia Ounoughi, «Monique De Mattia-Viviès, Leçons de grammaire anglaise, de la recherche à

l'enseignement », Lidil [En ligne], 62 | 2020, mis en ligne le 03 novembre 2020, consulté le 05 novembre 2020. URL : http://journals.openedition.org/lidil/8257 ; DOI : https://doi.org/10.4000/lidil.8257

Ce document a été généré automatiquement le 5 novembre 2020.

(c) Lidil 


\section{Monique De Mattia-Viviès, Leçons de grammaire anglaise, de la recherche à l'enseignement}

3 vol., Aix-en-Provence, Presses universitaires de Provence, coll. « Manuels », 2018 et 2019, 1807 p.

\section{Samia Ounoughi}

\section{RÉFÉRENCE}

Monique De Mattia-Viviès, Leçons de grammaire anglaise, de la recherche à l'enseignement, 3 vol., Aix-en-Provence, Presses universitaires de Provence, coll. « Manuels », 2018 et 2019, 1807 p.

1 L'auteure de ces trois volumes de grammaire-linguistique anglaise, Monique De MattiaViviès, est professeure des universités en linguistique anglaise à Aix-Marseille Université (AMU-LERMA). Au fil de ces quelques 1807 pages, elle offre aux étudiants, aux enseignants et aux chercheurs un outil précieux d'accompagnement à la description, l'explication et l'analyse des phénomènes de linguistique et de grammaire anglaise. Le premier volume en deux tomes est consacré à la syntaxe et porte un regard sur l'ensemble de l'énoncé (Syntaxe 1. Théorie, 2018, 576 p.; Syntaxe 2. Commentaires grammaticaux, 2018, 185 p.). Viennent ensuite deux volumes portant sur les constituants de la syntaxe : Groupe nominal $(2019,581$ p.) et Groupe prédicatif $(2019,465$ p.). Ces deux volumes comprennent également des commentaires grammaticaux à la suite de la partie théorique.

2 Issu d'années d'expérience en enseignement et en recherche, Leçons de grammaire anglaise se distingue par la démarche didactique que l'auteure propose, à commencer par une description et une explication de la méthodologie. Chaque volume se divise en parties elles-mêmes contenant des leçons qui renvoient aux commentaires proposés en fin de volume (Groupe nominal, Groupe prédicatif) ou dans le deuxième tome du volume 1 
(Syntaxe 2. Commentaires grammaticaux). Le plan est annoncé au début de chaque leçon. Pour chaque type de constituant de la langue abordé, l'auteure part d'une description élémentaire qui permet à chaque lecteur, quel que soit son niveau d'avancement dans la discipline, de commencer son étude sur des bases sûres. Très pédagogique, l'ouvrage ne présume jamais de ce qui est censé appartenir au déjà su et se propose d'accompagner le lecteur dès les premières années de licence. La complexification des explications se fait progressivement, avec patience, pourrait-on écrire. À l'intérieur de chaque leçon, on trouvera également une note synthétique qui permet de retrouver l'essentiel à la fin de chaque sous-partie et de naviguer plus efficacement dans le manuel. En outre, l'auteure propose des récapitulatifs sous forme de tableaux en plus des conclusions rédigées, ainsi que des modalités variées qui conviennent à des techniques et des méthodes d'apprentissage différentes chez les lecteurs.

Du point de vue scientifique, Leçons de grammaire anglaise se caractérise par sa grande ouverture et fait entrer en résonance différents courants de la linguistique et de la grammaire. Ainsi, la syntaxe est appréhendée par le biais de la grammaire transformationnelle et de la grammaire générative. La linguistique de l'énonciation et la TOPE culiolienne, la linguistique et la pragmatique concourent quant à elles à décrire et expliquer les phénomènes relatifs au groupe nominal et au groupe prédicatif. Soulignons enfin l'apport précieux des aspects de la linguistique dont l'auteure est une grande spécialiste, notamment sur le discours rapporté. La démarche qui consiste à mettre les "théories linguistiques en dialogue " (nous citons le titre de la série de colloques organisés par Laure Gardelle et Laurence Vincent-Durroux, UGA-LIDILEM) loin de compliquer les leçons, participe efficacement à l'enseignement de la méthode. Ainsi, face à un segment à commenter, l'étudiant, le candidat au concours envisagera des entrées multiples dans son étude dès lors enrichie. C'est cette même approche scientifique qui évite en outre un cloisonnement dans un courant de la linguistique avec des descriptions ou des explications qui trouveraient rapidement des limites. Au fil des leçons, le lecteur apprendra aussi à distinguer les plans différents et parfois contradictoires (plan sémantique, plan syntaxique, notamment) que les exemples peuvent présenter, pour apprendre à les discriminer, à les analyser et à les confronter. Le lecteur appréciera les enjeux d'une telle démarche en particulier dans ce que l'auteure nomme "la syntaxe mensongère ». Les constituants de la langue font l'objet d'une observation d'un fonctionnement en contexte et en situation pour éviter toute catégorisation définitive. Dans cette perspective, ce manuel constitue un véritable outil d'aide à la réflexion qui donne au lecteur les moyens de comprendre les réalités du langage au travail.

4 La variété et le nombre des exemples choisis, tant dans la langue courante que dans la littérature, concourent aussi à l'ouverture et à la volonté de montrer le fonctionnement de la langue en tenant compte de la créativité à laquelle elle donne lieu. Aux exemples singuliers s'ajoutent les résultats des analyses de corpora qui apportent des données quantitatives (sur la fréquence d'occurrences d'un agencement syntaxique, par exemple). Ces données offrent une vue d'ensemble sur les phénomènes à l'étude tout en gardant une fenêtre ouverte sur d'autres cas où le fonctionnement diffère. Enfin, les bibliographies de grammaire et de linguistique sont d'une grande richesse et sont une porte d'entrée très utile pour approfondir ses connaissances et mener des recherches sur les questions traitées dans l'ouvrage. Leçons de grammaire anglaise est le manuel de linguistique anglaise en français le plus complet depuis Linguistique et grammaire de l'anglais (Lapaire \& Rotgé, 1993). Il rappelle, renouvelle et complète ses 
prédécesseurs alliant subtilement recherche et enseignement. Il s'impose comme un outil incontournable pour les étudiants anglicistes et les candidats aux concours du CAPES et de l'agrégation d'anglais.

\section{AUTEURS}

\section{SAMIA OUNOUGHI}

LIDILEM (EA 609), Université Grenoble Alpes 